\title{
Consideraciones en redacción científica: discusión, conclusiones y referencias
}

\section{Considerations in scientific writing: discussion, conclusions and references}

\begin{abstract}
Resumen
La sección de discusión es el segmento encargado de interpretar y analizar los resultados de un estudio; así como presentar las implicancias prácticas/clínicas de los hallazgos, las limitaciones del estudio y recomendaciones para futuros estudios que busquen mejorar la investigación original. Al igual que los distintos segmentos de un artículo científico, las secciones de discusión, conclusiones y referencias bibliográficas siguen reglas estandarizadas de redacción y publicación científica que deben ser conocidas por los autores. Conocer estas reglas otorgará al autor la oportunidad de facilitar y agilizar el proceso de preparación y publicación de su manuscrito mientras que al lector le permitirá entender con mayor sencillez los datos encontrados en el estudio. En la presente nota científica se resumen las principales recomendaciones y guías para redactar apropiadamente la discusión, conclusiones y referencias bibliográficas de un artículo científico.
\end{abstract}

Palabras clave: Bibliografía; Comunicación y divulgación científica; Escritura médica; Odontología; Publicaciones de divulgación científica.

\begin{abstract}
The discussion section is responsible for interpreting and analyzing the results of a study, as well as presenting the practical/clinical implications of the findings, the limitations of the study and recommendations for future studies aimed to improve the original research. As the different segments of a scientific article, the discussion, conclusions and references follow standardized rules of scientific writing and publication that should be known by the authors. These rules will give the author the opportunity to facilitate and expedite the process of preparation and publishing. In the other hand, the reader will be able to understand, in a simple way, the data found in the study. This scientific note summarizes the main recommendations and guidelines for properly writing the discussion, conclusions and references of a scientific article.
\end{abstract}

Keywords: Bibliography; Dentistry; Medical writing; Publications for science diffusion; Scientific communication and diffusion.

\section{Nota Científica}

Yuri Castro-Rodríguez ${ }^{1, a}$, Manuel Antonio Mattos-Vela ${ }^{2, b}$, Aron Aliaga-Del Castillo ${ }^{3, c}$

${ }^{1}$ Pontificia Universidad Católica del Perú. Universidad Privada Juan Pablo II. Lima, Perú.

2 Universidad Nacional Mayor de San Marcos,

Facultad de Odontología. Lima, Perú.

${ }^{3}$ Universidade de São Paulo, Faculdade de

Odontologia de Bauru. São Paulo, Brasil.

a Magíster en Educación.

b Doctor en Estomatología

c Maestro en Ortodoncia.

\section{Correspondencia:}

Yuri Castro Rodríguez

Correo electrónico: yuricastro_16@hotmail.com

Jr. Tomás Catari 463. Urb. El trébol. Los Olivos. Lima, Perú.

Conflicto de intereses: Los autores declaran no tener conflictos de interés.

Fuente de financiamiento: Autofinanciado

Fecha de recepción: 22/10/18

Fecha de aceptación: 07/11/18

(C) Los autores. Este artículo es publicado por la revista Odontología Sanmarquina de la Facultad de Odontología, Universidad Nacional Mayor de San Marcos. Este es un artículo de acceso abierto, distribuido bajo los términos de la licencia Creative Commons Atribucion - No Comercia_Compartir Igual 4.0 Internacional. (http://creativecommons.org/licenses/by-nc-sa/4.0/) que permite el uso no comercial, distribución y reproducción en cualquier medio, siempre que la obra original sea debidamente citada. 


\section{Introducción}

La investigación biomédica involucra a los profesionales de las ciencias de la salud, pacientes, docentes y estudiantes. Como pacientes, deseamos ser tratados con la mejor opción disponible basada en evidencia científica de alta calidad. Como docentes queremos enseñar lo más actual y apropiado de la evidencia dentro de nuestro campo. Como estudiantes queremos aprender la información más relevante y útil para la práctica clínica, y como investigadores deseamos seguir los métodos más apropiados en cada uno de nuestros estudios con el objetivo de generar aportes de buena calidad que busquen resolver determinados problemas de nuestra sociedad.

Sin embargo, detectar la "mejor evidencia científica" requiere que los estudios sean publicados de forma precisa, completa, acorde a los principios de redacción y protocolos de producción científica. Aquellos artículos científicos que no se comunican de forma correcta se convierten en evidencias incompletas, poco confiables, además de resultar en una investigación desperdiciada ${ }^{1}$.

Un buen artículo científico combina el pensamiento ordenado, estructura lógica, descripción completa y precisa de los elementos clave del estudio así como sencillez y claridad al momento de escribirlo ${ }^{2}$. Por ello surgen los protocolos de redacción y comunicación científica (principios para comunicar ensayos clínicos, estudios observacionales, revisiones sistemáticas, reportes de caso, estudios de diagnóstico, entre otros tipos de estudio).

En los últimos segmentos de un artículo científico se plasman el significado y la interpretación de los resultados, se añaden las fuentes de información que soportan los aspectos teóricos de la investigación, se discuten los principales hallazgos del estudio y se brindan las conclusiones de la evidencia encontrada, basada en los objetivos inicialmente planteados.

La presente nota científica finaliza la serie de resúmenes expuestos por los autores sobre los principales aspectos a considerar durante la redacción de un manuscrito. Anteriormente se establecieron pautas para la redacción del título, resumen y palabras clave ${ }^{3}$; la introducción y los métodos ${ }^{4}$; los resultados, tablas y figuras ${ }^{5}$. En la presente nota científica se resumirán las principales pautas para redactar las secciones de discusión, conclusiones y referencias bibliográficas de un trabajo de investigación en el campo de la Odontología.

\section{La discusión}

Derivado del latín "discutere" que significa "disipar, zanjar o resolver”; en el campo de la investigación implica examinar de forma rigurosa y atenta una materia ${ }^{6}$.

Es el segmento que responde la pregunta ¿Qué significan los resultados?, presenta una naturaleza argumentativa que intenta convencer al lector sobre la validez de los hallazgos ${ }^{7}$. Es el "corazón" de un artículo científico, el lugar donde la mayoría de lectores críticos acudirán luego de leer el resumen; asimismo es la sección más compleja de elaborar y redactar, puesto que pone a prueba la fortaleza científica de una investigación ${ }^{8}$.

Al igual que los anteriores segmentos de un artículo científico debe ser claro, ordenado, conciso, siguiendo una secuencia lógica, para lo cual se hacen las siguientes recomendaciones (Tabla 1).

Los estudios que presentan una buena discusión de sus resultados suelen asegurar su publicación. Lo contrario

Tabla 1. Características y recomendaciones al momento de redactar una discusión

\begin{tabular}{|c|c|}
\hline Características & Recomendaciones para su redacción \\
\hline $\begin{array}{l}\text { Presenta las relaciones, generalizaciones y principios de los } \\
\text { resultados encontrados }{ }^{9} \text {. }\end{array}$ & $\begin{array}{l}\text { Esmérese: Es la parte que más peso e importancia tiene en un } \\
\text { artículo científico. No argumente muchos datos sin concluir algo } \\
\text { relevante }{ }^{9,10} \text {. }\end{array}$ \\
\hline $\begin{array}{l}\text { Analiza los resultados, se interpretan en relación a los avances de } \\
\text { la ciencia, los compara, discute y se confronta con los resultados } \\
\text { de otros estudios además de resaltar lo novedoso y relevante del } \\
\text { estudio }^{6} \text {. }\end{array}$ & $\begin{array}{l}\text { Explique el significado científico de los hallazgos: interprete los re- } \\
\text { sultados más importantes, relacionándolos con los objetivos; va más } \\
\text { allá de una simple descripción. Se deben analizar y comparar con } \\
\text { base en los conocimientos previos. }\end{array}$ \\
\hline $\begin{array}{l}\text { Indica las excepciones o aspectos no resueltos del problema } \\
\text { estudiado }{ }^{9} \text {. Presenta las limitaciones y las implicaciones de } \\
\text { los hallazgos que permitan desarrollar nuevas investigaciones } \\
\text { (recomendaciones). }\end{array}$ & $\begin{array}{l}\text { Relaciónelo con la hipótesis (en caso que se haya requerido): basado } \\
\text { en los resultados, se consigue aceptar o rechazar y discutir la hipóte- } \\
\text { sis planteada. }\end{array}$ \\
\hline $\begin{array}{l}\text { Presenta las consecuencias teóricas de los hallazgos así como sus } \\
\text { aplicaciones prácticas }{ }^{9} \text {. }\end{array}$ & $\begin{array}{l}\text { Siempre fundamentar la discusión de resultados en base a estudios } \\
\text { ya publicados. Evitar el uso de especulaciones sin base científica. }\end{array}$ \\
\hline $\begin{array}{l}\text { La interpretación de los resultados se realiza dentro de los límites } \\
\text { de la lógica del estudio. }\end{array}$ & 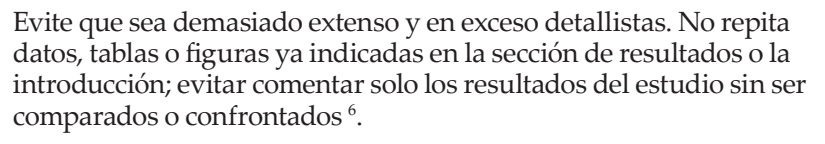 \\
\hline $\begin{array}{l}\text { Resalta las características más novedosas e importantes de los } \\
\text { resultados, así como su contextualización acorde a las evidencias } \\
\text { existentes }{ }^{11} \text {. }\end{array}$ & $\begin{array}{l}\text { Siga un orden lógico acorde a la presentación de resultados obte- } \\
\text { nidos, criterios metodológicos, variables y comparación con otros } \\
\text { estudios. }\end{array}$ \\
\hline $\begin{array}{l}\text { Los hallazgos son discutidos y no solamente repetidos, en los } \\
\text { casos de repeticiones se analizan los patrones }{ }^{12} \text {. }\end{array}$ & $\begin{array}{l}\text { Cuando se cite a algún autor o se indique alguna aseveración se debe } \\
\text { indicar la referencia. Se debe citar las fuentes de información que } \\
\text { fueron consultadas en su totalidad }{ }^{6} \text {. }\end{array}$ \\
\hline
\end{tabular}


sucederá con unos buenos resultados y una mala redacción de la discusión.

La sección de discusión se centra en establecer posibles explicaciones a los resultados encontrados, no necesariamente respaldando la hipótesis planteada. Además, se debe destacar las características novedosas y relevantes de la investigación, por qué el estudio tiene mérito para ser publicado, así como las conclusiones que derivaron de ella ${ }^{13}$. La interpretación se redacta en tiempo presente. Ejemplo: "Estos datos indican que...". Los resultados (del estudio realizado o de otros estudios) se redactan en tiempo pasado. Ejemplo: "Se halló una alta prevalencia de caries, lo cual concuerda con los resultados hallados por Castro..." (Los resultados van en pasado, la interpretación, el comentario o análisis va en tiempo presente).

Las limitaciones y las recomendaciones deben guardar relación entre sí; estas deben sugerir alternativas para realizar estudios salvando todas las limitaciones encontradas en la investigación original ${ }^{14}$. No es apropiado indicar solamente que "se requiere más estudios".

El Comité Internacional de Editores de Revista Médicas (ICMJE, por sus siglas en inglés) recomienda exponer las conclusiones y las recomendaciones dentro del segmento de la discusión; sin embargo, muchas revistas exigen que las conclusiones sean redactadas en un segmento separado ${ }^{13}$.

Al redactar la discusión, los autores no deben reflejar sus emociones personales, pues sería un texto sesgado y muchas veces demasiado amplio. Se recomienda siempre explicar y discutir ideas basadas en hechos reportados anteriormente y no sólo basarse en especulaciones. Se sugiere además finalizar comentando la importancia de los hallazgos encontrados así como la implicancia práctica y/o clínica del estudio.

Los artículos originales, revisiones sistemáticas y reportes de caso suelen ser los principales tipos de artículos científicos que presentan la sección de discusión (Tabla 2).

La pauta STROBE ${ }^{14}$ recomienda que para estructurar los apartados de la discusión se pueden utilizar incluso subtítulos: Resultados principales (comenzar la discu- sión con un breve resumen de los principales resultados encontrados). Limitaciones (considerar las fuentes de sesgo e imprecisiones que puedan afectar los resultados, así como la "importancia" relativa que tienen estos sesgos). Interpretación (considerando los objetivos, limitaciones, análisis de multiplicidad, resultados de estudios similares y otras evidencias relevantes, asimismo responder a las preguntas ¿Cuán fuerte es la asociación con la exposición? ¿Procedió la exposición al inicio de la enfermedad? ¿La asociación es consistente con observaciones en diferentes estudios y contextos? ¿Hay evidencia que apoye los hallazgos provenientes de estudios experimentales, sean de laboratorio o animales? ¿Cuán específica es la exposición para el efecto que se le imputa y existe una relación dosis-respuesta? ¿La asociación es biológicamente plausible? Generabilidad (indicar el grado en que los hallazgos se pueden aplicar a otras situaciones y responde a las preguntas: ¿Es posible aplicar los hallazgos a grupos o individuos que difieran de aquellos en donde se realizó el estudio? Usualmente es una cuestión de juicio que depende del contexto de la investigación, características de los estudiados, exposiciones estudiadas y los desenlaces.

Para ensayos clínicos la pauta CONSORT ${ }^{17}$ indica que las limitaciones deben abordar: limitaciones del ensayo, fuentes de posibles sesgos, imprecisiones y si fuese relevante, análisis de multiplicidad. Mientras que el abordaje de la generabilidad de los datos debe incluir: validez externa y aplicabilidad de los resultados. Asimismo, la interpretación de los datos debe ser coherente con los resultados, equilibrar los beneficios y los daños, considerar otras evidencias relevantes.

\section{Las conclusiones}

Segmento que señala la interpretación general de los hallazgos acorde a otras evidencias, así como sus repercusiones para estudios futuros; responde a la pregunta formulada en la introducción, a los objetivos, y son apropiadas para los resultados obtenidos ${ }^{13}$.

Es frecuente que se fusionen con la sección de discusión; sin embargo, recomendamos revisar las directrices para los autores de una determinada revista para revisar este punto. Algunas de sus características y recomendaciones para su redacción se resumen en la tabla 3.

Tabla 2. Características de la discusión según tipo de artículo científico

\begin{tabular}{|c|c|c|}
\hline Artículos originales & Reportes de caso & Revisiones sistemáticas \\
\hline $\begin{array}{l}\text { Una estructura típica incluiría: breve } \\
\text { presentación de los principales resultados; } \\
\text { valoración de las fortalezas y debilidad } \\
\text { del estudio; comparación de los resultados } \\
\text { con estudios previos; consideraciones e } \\
\text { implicancias de los hallazgos; limitaciones } \\
\text { del estudio y recomendaciones para futuros } \\
\text { estudios }{ }^{7} \text {. }\end{array}$ & $\begin{array}{l}\text { Describir las fortalezas y debilidades en el } \\
\text { manejo del caso, discutir la literatura mé- } \\
\text { dica pertinente, justificar las conclusiones } \\
\text { y presentar las principales lecciones que se } \\
\text { pueden extraer del caso }{ }^{15} \text {. } \\
\text { Limitar la cantidad de citas y centrarse en } \\
\text { las más relevantes. }\end{array}$ & $\begin{array}{l}\text { Resumir los hallazgos principales, incluir } \\
\text { el nivel de evidencia para cada hallazgo } \\
\text { principal. Considerar la relevancia de los } \\
\text { hallazgos para cada grupo de personas (los } \\
\text { que brindan tratamientos, los usuarios y los } \\
\text { responsables políticos). Discutir limitacio- } \\
\text { nes relacionadas al estudio, a los resultados } \\
\text { encontrados (riesgo de sesgo) y al proceso } \\
\text { de revisión (recuperación incompleta de } \\
\text { investigaciones encontradas o sesgo de } \\
\text { reporte de información) }{ }^{16} \text {. } \\
\text { Suele tener una amplia cantidad de citas } \\
\text { bibliográficas. }\end{array}$ \\
\hline
\end{tabular}


Tabla 3. Características y recomendaciones al momento de redactar las conclusiones

\begin{tabular}{|c|c|}
\hline Características & Recomendaciones \\
\hline Deben guardar relación con los objetivos del estudio ${ }^{13}$. & $\begin{array}{l}\text { Evitar aseveraciones relacionadas a los beneficios y costos económicos a } \\
\text { menos que sean parte de los resultados }{ }^{13} \text {. }\end{array}$ \\
\hline $\begin{array}{l}\text { Se redactan en pasado. } \\
\text { No deben ser imprecisas o abstractas. }\end{array}$ & Evite expresiones como: podría ser que, eventualmente... \\
\hline Cualquier conclusión debe ser respaldada por los resultados del estudio ${ }^{7}$. & $\begin{array}{l}\text { Evitar prioridades entre las conclusiones y no establecer conclusiones prema- } \\
\text { turas de estudios que se encuentran en curso }{ }^{13} \text {. }\end{array}$ \\
\hline $\begin{array}{l}\text { Debe contemplar tres aspectos: presentar los hechos comprobados en el } \\
\text { estudio, redacción clara y concisa, ser ordenadas acorde a los objetivos. }\end{array}$ & $\begin{array}{l}\text { Estructure de tal forma que resalte el estudio }{ }^{10} \text { : centrarse en la hipótesis, } \\
\text { punto fuerte y originalidad del estudio. Presente las consecuencias más } \\
\text { relevantes. }\end{array}$ \\
\hline $\begin{array}{l}\text { Deben responder a la formulación del problema planteada en la introducción. } \\
\text { Permite condensar las deducciones, consecuencias o resoluciones en torno a } \\
\text { los objetivos, hipótesis, marco teórico, resultados y análisis de datos }{ }^{10} \text {. }\end{array}$ & $\begin{array}{l}\text { Puede ser el último párrafo de la discusión en los casos que se exija artículos } \\
\text { cortos. Puede ser largo cuando se exija artículos largos }{ }^{10} \text {. }\end{array}$ \\
\hline $\begin{array}{l}\text { La concisión es la cualidad que caracteriza esta sección; procede de un rigu- } \\
\text { roso proceso deductivo y mesurado sin que interfieran las dudas razonables } \\
\text { del estudio. }\end{array}$ & $\begin{array}{l}\text { Evitar que presenten afirmaciones que no fueron contrastadas o que no } \\
\text { fueron lo suficientemente respaldadas por los datos obtenidos }{ }^{13} \text {. }\end{array}$ \\
\hline $\begin{array}{l}\text { Es diferente a la del resumen pues resalta los puntos más relevantes de los } \\
\text { hallazgos y es más extenso }{ }^{10} \text {. }\end{array}$ & $\begin{array}{l}\text { Evitar la repetición de datos ya mencionados en los resultados y discusión o } \\
\text { aquellos que no se relacionan con el estudio. }\end{array}$ \\
\hline Carece de especulación o ausencia de debate académico ${ }^{10}$. & No agregar referencias pues pone en duda su trabajo y le quita importancia ${ }^{10}$. \\
\hline
\end{tabular}

\section{Las referencias}

También denominada: "Referencias bibliográficas", "Referencias de consulta", "Bibliografía". Para el ICMJE lo apropiado es: Referencias ${ }^{13}$.

Es la sección que expone las fuentes de la información utilizadas para redactar el manuscrito. Estas fuentes pueden ser verificadas por los lectores; por tal motivo su elección debe ser cuidadosa y justificada ${ }^{6}$, sin llegar al extremo de incluir muy pocas que sean insuficientes para sustentar las aseveraciones o ser tan numerosas.

Permite otorgarles validez y calidad a los escritos ya que deben estar respaldados por referencias de consulta que sustentan o ratifican conceptos vertidos en el contenido del artículo ${ }^{6}$. Reflejan la totalidad de la fuente referida a las citas bibliográficas.

En el campo biomédico se emplea generalmente el estilo Vancouver seguido del estilo Harvard; sin embargo, no deben ser mezclados y debe seguirse el que la revista específica exija ${ }^{10}$.

Es importante tener presente algunas pautas para su elaboración (Tabla 4).

Seleccionarlas está en plena libertad de los autores; sin embargo se debe tener ciertas consideraciones ${ }^{13}$ :

- Deben ser accesibles para su verificación tanto por parte de los editores, revisores y lectores.

- Mantener una concordancia con el tema expuesto.

- Evitar incluir un exceso de citas con conceptos vagos o poco relacionados con el tema.

- Deben ser mencionados de forma secuencial, es decir, numerarse de acuerdo a su aparición en el texto (Vancouver) (algunos lo prefieren en orden alfabético). Pero eso dependerá finalmente del estilo de la revista.

- Deberán ser justificadas con una respectiva aseveración o idea.

- Deben ser un complemento para que el lector aumente sus conocimientos sobre el tema.

- Evitar comunicaciones personales o de expertos a no ser que se considere de amplio reconocimiento y sea información trascendental, en tal caso colocar el nombre y la fecha entre paréntesis: Ejemplo: (Castro $Y, 2009$ )

- Las referencias no deben ser utilizadas por autores, editores o revisores para promover intereses propios.

- Aunque las referencias a artículos de revisión pueden ser una manera eficiente de guiar a los lectores a las fuentes de información. Estos artículos no siempre reflejan el trabajo original con precisión por lo que es preferible buscar las fuentes originales.

- Referencias a trabajos aceptados pero aún no publicados. deben ser designados como "en prensa" "in press" o "de próxima aparición". Asimismo, información de manuscritos enviados pero no aceptados deben ser citados en el texto como "observaciones no publicadas" con permiso por escrito de la fuente.

- Los autores son responsables de verificar que ninguna de las referencias cite a artículos retractados, excepto en el contexto de referencia a la retractación. Para artículos publicados en revistas indexadas en MEDLINE, el ICMJE considera a PubMed como la fuente autorizada de información sobre retractaciones. Los autores pueden identificar artículos retirados en MEDLINE buscando en PubMed: "Pu- 
blicación retirada $[\mathrm{pt}]$ ", donde el término "pt" entre corchetes significa tipo de publicación, o yendo directamente a la lista de PubMed de publicaciones retiradas.

- Dependiendo del tipo de artículo a escribir se debe seleccionar el número de referencias apropiadas, en reportes de caso suelen ser entre 10-15, en artículos originales entre 15-25, mientras que en artículos de revisión más de 30 (aunque depende de cada revista, esta es sólo una recomendación).

Es común que los editores de revistas científicas encuentren la mayor cantidad de errores de redacción en este segmento; en la figura se presentan modelos generales de referencias acorde a las directrices del estilo Vancouver así como errores frecuentes al momento de su redacción. Para una visualización de más ejemplos de citas y referencias bibliográficas recomendamos las guías y directrices de la "U.S National Library of Medicine": https://www.nlm.nih.gov/bsd/uniform_requirements. html\#journals.

Recordamos que el formato final de referencias dependerá de las recomendaciones dadas por cada revista en particular.

\section{Conclusiones}

Las últimas secciones de un manuscrito permiten a los autores presentar, interpretar y discutir los hallazgos más importantes; así como sintetizar las fuentes de información utilizadas. Un análisis cuidadoso y bien informado del significado de los hallazgos del estudio y su posible influencia en el área de estudio es crucial para mejorar la redacción de estas últimas secciones, garantizando la publicación del estudio y aumentando su visibilidad e impacto.

\section{A}

No colocar las iniciales
de los nombres

1. Tadic, Beckmann, Schwarz Kirko, Epple M. A novel method to produce hydroxyapatite objects with interconnecting porosity that avoids sintering. Biomaterials. 2004; 25:3335-40.

$$
\begin{gathered}
\text { No colocar a todos los } \\
\text { autores }
\end{gathered}
$$

2. Chai YC, et al. Current views on calcium phosphate osteogénicas and the translation into effective bone regeneration strategies. Acta de Biomateriales 2012;8(11):3876-87.

$$
\text { No abreviar la revista } \quad \begin{gathered}
\text { No colocar un punto entre } \\
\text { la revista y el año }
\end{gathered}
$$

3. Barrera F, van Blitterswijk CA, de Gr"oot K. Bone regeneration: molecular and cellular interactions with calcium phosphate ceramics. Int J Nanomedicine. 2006;1(3):317.

$$
\begin{gathered}
\text { Errores en la redacción de } \\
\text { los apellidos }
\end{gathered}
$$$$
\text { No colocar las páginas }
$$

4. Salinas AJ, Vallet Regí M. Bioactive ceramics: from bone grafts to tissue engineering. RSC Adv. 2013;3(28):11116-31.

$$
\text { Redactar mal los apellidos }
$$

$$
\text { compuestos }
$$

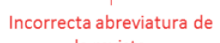

$$
\text { la revista }
$$

B $\quad \begin{array}{r}\text { Colocar los seis primeros } \\ \text { autores, seguidos de }\end{array}$

. Sivakumar M, Kumar TSS, Shantha KL, Rao KP, Neman L, Carls R, et al. Development of hydroxyapatite derived from Indian coral. N Engl J Med. 2002 Jul 25;347(4):284-7.

Colocar la abreviatura de la revista, seguida de un puntoy el año de publicación

Opcional: Si una revista lleva una paginación continua a lo largo de un volumen (como hacen muchas revistas médicas), omita el mes y el número de emisión

2. Van Halpern SD. Solid-organ transplantation in HIV-infected patients. N Engl J Med. 2002;347:284-7.

$$
\begin{aligned}
& \text { Respetar los títulos y apellidos compuestos } \\
& \text { de los autores }
\end{aligned}
$$

3. Salinas-Castro AJ, Vallet-Regí M. Bioactive ceramics: from bone grafts to tissue engineering. PubMed PMID: 19204236; PubMed Central PMCID: PMC2653214.

$$
\begin{aligned}
& \text { Opcional: Añadir los identificadores únicos de una base de datos, como el } \\
& \text { PMID o el DOI }
\end{aligned}
$$

4. Blokhuis TJ, Termaat MF, den Boer FC, Patka P, Bakker FC, Haarman HJ. Properties of calcium phosphate ceramics in relation to their in vivo behavior. J Trauma. 2000;48(1):179-86. doi: 10.1111/cid.12682.

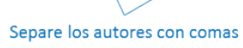

Seguir el modelo del uso del punto y coma, paréntesis y dos puntos. No utilizar espacios de separación entre cada signo

5. Trachtenberg F, Maserejian NN, Soncini JA, Hayes C, Tavares M. Does fluoride in compomers prevent future caries in children? 2002;42 Suppl 2:S93-9. ClinicalTrials.gov registration number: NCT00065988.

$$
\text { se colocará paréntesis }
$$

Figura. A. Errores frecuentes al redactar una referencia. B. Ejemplos de referencias correctamente redactadas de acuerdo al estilo Vancouver 


\section{Referencias bibliográficas}

1. Breugelmans JG, Roberge G, Tippett C, Durning M, Struck DB, Makanga MM. Scientific impact increases when researchers publish in open access and international collaboration: A bibliometric analysis on poverty-related disease papers. PLoS One. 2018;13(9):e0203156. doi: 10.1371/journal.pone.0203156. eCollection 2018.

2. Junaid J. How to submit to a scientific journal and get published. J Pak Med Assoc. 2012;62(9):977-8.

3. Castro-Rodríguez Y, Mattos-Vela MA, Aliaga-Del Castillo A. Consideraciones en redacción científica: el título, resumen y palabras clave. Odontol Sanmarquina. 2018; 21(1): 63-6. http://dx.doi.org/10.15381/os.v21i1.14431

4. Castro-Rodríguez Y, Mattos-Vela MA, Aliaga-Del Castillo A. Consideraciones en redacción científica: la introducción y los métodos. Odontol Sanmarquina. 2018;21(2):14752. http://dx.doi.org/10.15381/os.v21i2.14781

5. Castro-Rodríguez Y, Mattos-Vela MA, Aliaga-Del Castillo A. Consideraciones en redacción científica: los resultados, tablas y figuras. Odontol Sanmarquina. 2018;21(3):2418. http://dx.doi.org/10.15381/os.v21i3.15129

6. Aranda TE, Mitru TN, Costa AR. ABC de la redacción y publicación médico-científica. En: Mitru TN. El título del artículo. 2da ed. Madrid: Cooperación Cultural Exterior; 2009. p. 61-70.

7. Simera I, Altman DG. Reporting medical research. Int J Clin Pract. 2013;67(8):710-6. doi: 10.1111/ijcp.12168.

8. Villagran AT, Harris PR. Algunas claves para escribir correctamente un artículo científico. Rev Chil Pediatr. 2009;80(1):70-8.

9. Day RA. Cómo escribir y publicar trabajos científicos. 3era ed. Washington: Organización Panamericana de la Salud-OPS; 2005.

10. Cohen C. Guía de redacción científica. CIRAD 2013. [Consultado el 10 de marzo del 2018] Disponible en: https://coop-ist.cirad.fr/actualites/guia-de-redaccion-cientifica
11. Comité Internacional de Editores de Revistas Médicas. Requisitos de Uniformidad para los manuscritos enviados a revistas biomédicas: escritura y proceso editorial para la publicación de trabajos biomédicos. Rev Esp Cardiol. 2004;57(6):538-56.

12. Weinberger CJ, Evans JA, Allesina S. Ten Simple (Empirical) Rules for Writing Science. PLoS Comput Biol. 2015;11(4):1-6. https://doi.org/10.1371/journal. pcbi. 1004205

13. International Committee of Medical Journal Editors. Recommendations for the conduct, reporting, editing, and publication of scholarly work in medical journals (updated december 2017). [Consultado el 10 de octubre del 2018]. Disponible en: http://www.icmje.org/recommendations/.

14. Vandenbroucke JP, Von Elm E, Altman DG, Gøtzsche PC, Mulrow C, Pocock SJ et al. Mejorar la comunicación de estudios observacionales en epidemiología (STROBE): explicación y elaboración. Gac Sanit. 2009;23(2):158e1-158e28. http://dx.doi.org/10.1590/ S0213-91112009000200015.

15. CARE. Case report guidelines. [Consultado el 10 de octubre del 2018]. Disponible en: http://www.care-statement. org

16. Moher D, Liberati A, Tetzlaff J, Altman DG, The PRISMA Group. Preferred Reporting Items for Systematic Reviews and Meta-Analyses. PLoS Med. 2009;6(7): e1000097. doi:10.1371/journal.pmed1000097

17. Moher D, Hopewell S, Schulz KF, Montori V, Gøtzsche PC, Devereaux PJ, et al. CONSORT 2010 explanation and elaboration: updated guidelines for reporting parallel group randomised trials. Int J Surg. 2012;10(1):2855. doi: 10.1016/j.ijsu.2011.10.001. 